\title{
BMJ Open Contribution of serum lipids as effect modifiers to a relationship between mean arterial pressure and coronary heart disease in Chinese rural population: the Henan Rural Cohort Study
}

Xia Zhang, ${ }^{1}$ Yuqian $\mathrm{Li},{ }^{2}$ Yanhua Wang,${ }^{3}$ Kai Hu, ${ }^{4}$ Runqi Tu, ${ }^{1}$ Haiqing Zhang, ${ }^{1}$ Zhongyan Tian, ${ }^{1}$ Dou Qiao, ${ }^{1}$ Gongyuan Zhang, ${ }^{1}$ Chongjian Wang (D) ${ }^{1}$

To cite: Zhang X, Li Y, Wang Y, et al. Contribution of serum lipids as effect modifiers to a relationship between mean arterial pressure and coronary heart disease in Chinese rural population: the Henan Rural Cohort Study. BMJ Open 2019;9:e029179. doi:10.1136/ bmjopen-2019-029179

- Prepublication history for this paper is available online. To view these files, please visit the journal online (http://dx.doi. org/10.1136/bmjopen-2019029179).

$\mathrm{XZ}$ and $\mathrm{YL}$ contributed equally.

Received 15 January 2019 Revised 20 September 2019 Accepted 24 September 2019

Check for updates

(C) Author(s) (or their employer(s)) 2019. Re-use permitted under CC BY-NC. No commercial re-use. See rights and permissions. Published by BMJ.

For numbered affiliations see end of article.

Correspondence to Dr Chongjian Wang; tjwcj2005@126.com

\section{ABSTRACT}

Objectives The aim of this study was to evaluate the relationship between mean arterial pressure (MAP) and coronary heart disease (CHD) in Chinese rural population. In addition, we hypothesised that this relationship might be mediated by some degree of serum lipids.

Design This is a cross-sectional study.

Setting The participants were from the Henan Rural Cohort Study, initiated in five rural areas (Tongxu county of Kaifeng city, Yima county of Sanmenxia city, Suiping county of Zhumadian city, Xinxiang county of Xinxiang city and Yuzhou county of Xuchang city) in Henan Province, China, during July 2015 and September 2017.

Participants The study included 39020 subjects aged 18-79 years as current research population.

Outcome measures Systolic blood pressure (SBP) and diastolic blood pressure (DBP) were measured using an electronic sphygmomanometer. MAP was calculated by one-third SBP plus two-thirds DBP. The study used restricted cubic splines and logistic regression models to evaluate the ORs and 95\% Cls. Mediation analysis using bootstrap was performed to examine the contribution of serum lipids to MAP-related CHD.

Results The adjusted OR $(95 \% \mathrm{Cl})$ for the highest MAP quartile with the risk of CHD was 1.45 (1.24 to 1.69) compared with the lowest quartile. Simultaneously, each 1-SD increment in MAP was significantly associated with a $12 \%$ increased risk of CHD. A linear dose-response relationship between MAP and CHD was found ( $p$ value for non-linear $=0.1169$ ) in the fully adjusted model. We further reported that $36.07 \%$ of proportion explained risk of $\mathrm{CHD}$ was mediated through serum lipids.

Conclusions Increased MAP was a significant marker of CHD in Chinese rural population. Meanwhile, the relationship was mediated by some degree of serum lipids, and triglyceride was the strongest mediator.

Trial registration number Henan Rural Cohort study has been registered at Chinese Clinical Trial Register (ChiCTR-00C-15006699) and the stage it relates to is Post-results.

\section{Strengths and limitations of this study}

Focusing on the rural population includes the relatively large sample size.

- The present study had rigorous design and various statistical methods.

- The first one to explore the association was mediated by some degree of serum lipids.

- The causal association between mean arterial pressure and coronary heart disease was not established.

\section{INTRODUCTION}

Cardiovascular diseases (CVDs) are the enormous challenge for sustainable human development. ${ }^{1}$ Globally, the number of deaths caused by CVD has been increased from 12.59 million in 1990 to 17.92 million in $2015 .^{2}$ In China, there was around $42 \%$ increase within 25 years. ${ }^{3}$ It has been reported that coronary heart disease (CHD) accounts for a large proportion of all CVD cases. ${ }^{2}$ According to the 'China Health and Family Planning Statistical Yearbook 2016', in 2015, the mortality of CHD has increased significantly in rural areas, which was slightly higher than that in urban areas by $2015 .{ }^{4}$

A number of studies have proven that blood pressure (BP) is a widely accepted risk factor for CVDs. ${ }^{56}$ However, studies on BP as a risk factor for CHD have been concentrated on evaluating the effect of systolic blood pressure (SBP) and diastolic blood pressure (DBP), which only represent extreme conditions of pressure fluctuations. ${ }^{78}$ Moreover, mean arterial pressure (MAP) is a major component of $\mathrm{BP}$, which provides a comprehensive description of BP. ${ }^{9}$ Several previous studies 
have suggested the close relationship between MAP and CHD mortality. ${ }^{10-12}$ However, it has not been confirmed whether increased MAP is a significant marker of CHD in Chinese rural population .

Therefore, the present study aimed to evaluate the relationship between MAP and CHD in Chinese rural population. Furthermore, we hypothesised that this relationship might be mediated by some degree of serum lipids since multiple evidence supported the relationships of serum lipids with both $\mathrm{MAP}^{1314}$ and CHD. ${ }^{15-18}$

\section{MATERIALS AND METHODS Study subjects}

The study population was from the Henan Rural Cohort Study. The Henan Rural Cohort Study was investigated in five rural areas of Henan from July 2015 to September 2017, which has been registered in Chinese Clinical Trial Register (Registration No. ChiCTR-OOC-15006699) before the onset of patient enrolment. Sampling methods have been described in detail in the previous article. ${ }^{19}$ The subjects were excluded if they did not have the data of BP $(n=48)$ and serum lipids $(n=191)$. Finally, a total of 39020 eligible subjects were included for analysis. Written informed consent was acquired from each participant prior to data collection.

\section{Ethical considerations}

The purpose and the importance of the study were explained to the participants. Participants' personal identifiers were removed from the data collection questionnaire to maintain confidentiality of the information throughout the study.

\section{Patient and public involvement}

Neither patients nor the public were involved in the development of the project.

\section{Assessment of MAP}

According to the American Heart Association's standardised protocol, ${ }^{20}$ each participant who was required to sit at least $5 \mathrm{~min}$ had their SBP and DBP measured three times by using electronic sphygmomanometer (Omron HEM-7071A, Japan). The average of three readings was taken for the analysis. If a difference of more than $5 \mathrm{~mm} \mathrm{Hg}$ was observed, the two closest values were used for the average. To get accurate readings, the participants were asked to refrain from tea and alcohol consumption, cigarette smoking or excessive physical activity for at least 30 min or more before the measurement. MAP was calculated from one-third SBP plus two-thirds DBP.

\section{Assessment of potential covariates}

The data collected included basic information such as social and demographic characteristic, as well as health and lifestyle details, as described previously. ${ }^{21}$ Briefly, anthropometric parameters were measured twice and the average readings were used for statistical analysis. Weight and height (with light clothes and shoes off) were measured using standard measuring equipment to the nearest $0.1 \mathrm{~kg}$ and $0.1 \mathrm{~cm}$, respectively. The body mass index (BMI) was calculated on the basis of the height and weight measurements. Waist circumference (WC) was measured at the midpoint between the lowest rib and the iliac crest to an accuracy of $0.1 \mathrm{~cm}$.

\section{Definition of mediators}

After fasting overnight for at least 8 hours, venous blood specimen was collected in vacuum tubes without anticoagulation. Serum samples were isolated from whole blood by centrifugation at $3000 \mathrm{rpm}$ for $10 \mathrm{~min}$ at room temperature and then were sent to measure total cholesterol (TC), triglycerides (TG), high-density lipoprotein cholesterol (HDL-C) and low-density lipoprotein cholesterol (LDL-C) by Roche Cobas C501 automatic biochemical analyser. The measurement methods of TC, TG, HDL-C and LDL-C were cholesterol oxidase method, enzymatic method and the direct method, respectively.

\section{Definition of CHD}

All respondents were covered by the New Rural Cooperative Medical System (NRCMS), and each participant had a unique medical insurance card number and ID, making it easy to track disease incidence and mortality. The CHD from the epidemiological questionnaire was further identified and confirmed through the NRCMS medical records reviews by the outcome committee consisting of an internist, an endocrinologist, a cardiologist and an epidemiologist based on uniform and standardised diagnostic criteria. We defined CHD prevalence at the first hospital admission with an occurrence of an angina pectoris (International classification of diseases, ICD-10 code I20), acute myocardial infarction (I21), subsequent myocardial infarction (I22), other forms of acute (I24) or chronic (I25) heart disease, percutaneous transluminal coronary angioplasty or coronary artery bypass graft, and cardiac arrest (I46) or death with CHD (I20-I25) as the underlying cause. ${ }^{22}$

\section{Statistical analysis}

Characteristics of the participants were described as mean $\pm \mathrm{SD}$ and median $(\mathrm{IQR})$ for continuous variables and frequencies (percentages) for categorical variables. We used a one-factor analysis of variance or a $\chi^{2}$ test to analyse the relationship of MAP category with the other values. The age-standardised prevalence of CHD was calculated based on data from China's sixth census.

Logistic regression models were used to evaluate the relationship of the MAP quartiles and per SD increment of MAP with the risk of CHD after adjusting for potential confounders (age, gender, smoking, drinking, physical activity, family history of CHD, antihypertensive, lipid-lowering treatments and type 2 diabetes mellitus). In addition, the dose-response relationship between continuous MAP and the risk of CHD was assessed by the restricted cubic splines in logistic regression recommended according to Loic Desquilbet and François 
Mariotti ${ }^{23}$ using three knots located at 25th, 50th and 75th percentiles of MAP in the light of the distribution, with $74 \mathrm{~mm} \mathrm{Hg}$ (approximate the first knot) as the reference group. Finally, the contribution of serum lipids (including TC, TG, LDL-C and HDL-C) to MAP-related CHD was calculated by mediation analysis. The mediation analysis using PROCESS in SPSS has been introduced in the previous article. ${ }^{24}{ }^{25}$ Briefly, first of all, the total effect must be significant to ensure the conduct of mediation. Second, complete mediation exists when statistical difference was found in indirect effect but not in direct effect. Partial mediation exists when indirect and direct effects are both significant. The proportion caused by mediator is computed with the formula (indirect effect/total effect). Considering the skewed distribution of serum lipids including TC, TG, HDL-C and LDL-C, logarithmic transformations were used for all four indicators in the current study.

The statistical analyses were performed using IBM SPSS Statistics for Windows software, V.21.0 (IBM Corp, Armonk, New York, USA), SAS V.9.1 (SAS Institute, Cary, New Carolina, USA) and R V.3.5.0 (The R Foundation for Statistical Computing, Vienna, Austria). P value $<0.05$ was considered as statistically significant.

\section{RESULTS}

\section{Demographic characteristics of the participants}

The basic characteristics of the population were shown in table 1. Among the 39020 participants (15353 male and 23667 female), the mean age was 55.58 years old. Overall, 1716 participants were diagnosed with CHD. The crude and age-standardised prevalence of CHD were $4.40 \%$ and $2.23 \%$, respectively. MAP was increased in accordance with increased age. In addition, higher levels of WC, BMI, SBP, DBP, PP, TC, TG and LDL-C were along with the increase of MAP, while HDL-C decreased with an increase in MAP.

\section{Association between MAP and CHD}

Table 2 shows that MAP was associated with CHD in three models. In the unadjusted model, compared with the first quartile of MAP, the OR $(95 \% \mathrm{CI})$ of the highest quartile was 1.63 (1.41 to 1.88$)\left(\mathrm{P}_{\text {trend }}<0.0001\right)$. When MAP was analysed as a continuous variable, the OR (95\% CI) for the risk of CHD was 1.14 (1.09 to 1.19) for per 1-SD increment. In the fully adjusted model, the corresponding ORs (95\% CIs) were 1.38 (1.20 to 1.60$)$ and 1.09 (1.04 to $1.15)$, respectively.

In addition, the restricted cubic splines demonstrated that the risk of CHD increased gradually with continuous MAP. In the unadjusted model, the test for the overall association between MAP and CHD was significant ( $p$ value for overall $<0.0001)$. The non-linear relationship test demonstrated that this dose-response relationship was non-linear ( $p$ value for non-linear $=0.0002$ ). In the fully adjusted model, the test for the overall association between MAP and CHD was significant ( $p$ value for overall $=0.0228)$. The linear association test demonstrated that this dose-response relationship was linear ( $p$ value for non-linear=0.1169) (figure 1).

\section{Mediators of the effect of MAP on CHD}

The normal distribution tests were conducted in the variables (TC, TG, HDL-C and LDL-C), and the abnormal distribution tests were applied to analyse the data after logarithm transition. In mediation analysis, we found that the relationship of MAP-related CHD was mediated by some degree of serum lipids (TC, TG, HDL-C, LDLC). The results showed that the OR (95\% CI; p value) of total effect was 1.0113 (1.0078 to $1.0148 ; 0.001)$. The log-transformed value of TG had an indirect-effect OR of 1.0090 (1.0055 to 1.0126 ), followed by the logtransformed value of HDL-C, which had an indirect-effect OR of 1.0004 (1.0001 to 1.0007) (figure 2). We did not find that the log-transformed values of TC and LDL-C mediated significantly the relationship between MAP and CHD (data were not show).

In addition, previous studies have shown that TG/ HDL-C was also associated with CHD, and we further analysed the mediating effect of TG/HDL-C on MAP-CHD and found the indirect-effect OR of 1.0012 (1.0008 to $1.0015)$. When combining the three mediators, the total indirect-effect OR was still 1.0113 (1.0078 to 1.0148). In order to better distinguish the relative sizes of the mediation paths, the sizes of the three mediation routes were compared. The data showed that the mediating effect of the log-transformed value of TG was significantly higher than that of the log-transformed value of HDL-C (OR $1.0042 ; 95 \%$ CI 1.0030 to $1.0055 ; \mathrm{p}<0.05)$ and TG/HDL-C (OR 1.0054; 95\% CI 1.0034 to $1.0072 ; \mathrm{p}<0.05$ ). The $\log$ transformed value of HDL-C was also significantly higher than that of TG/HDL-C (OR 1.0012; 95\% CI 1.0001 to 1.0023; $\mathrm{p}<0.05)$.

The log-transformed value of TG explained the largest proportion explained risk of CHD. The proportion explained risk mediated was $21.43 \%$ for the logtransformed value of TG, 3.57\% for the log-transformed value of HDL-C and $19.67 \%$ for TG/HDL-C. Therefore, the log-transformed value of TG was the strongest mediator of the MAP-CHD relationship.

\section{DISCUSSION}

The present large survey specialised of Chinese rural population provided important new evidence for the significant relationship between MAP and CHD. A linear dose-response relationship between MAP and CHD was first observed in Chinese rural population. In addition, this relationship was mediated by some degree of TG, HDL-C and TG/HDL-C, and TG was the strongest mediator of the MAP-CHD relationship.

The results from this study showed that individuals with MAP $\geq 103 \mathrm{~mm} \mathrm{Hg}$ were 1.19-fold chance to develop CHD than those with MAP $<84 \mathrm{~mm} \mathrm{Hg}$, after adjusting for confounding factors. Our finding of a significantly 
Table 1 Characteristics of the participants according to quartiles of MAP

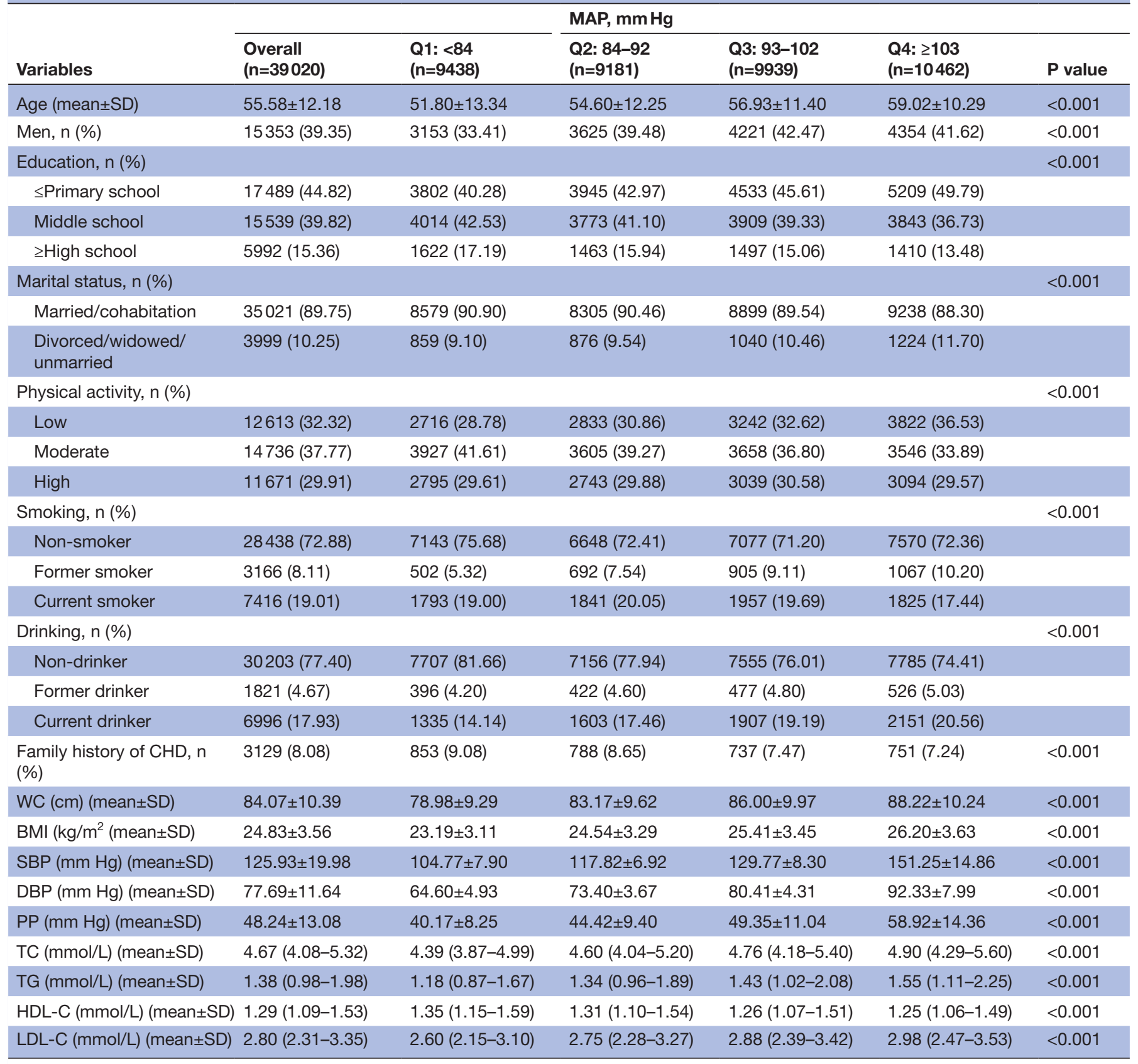

Data are mean $\pm \mathrm{SD}$, median (IQR) or $\mathrm{n}(\%)$.

BMI, body mass index;CHD, coronary heart disease; DBP, diastolic blood pressure;HDL-C, high-density lipoprotein cholesterol; LDL-C, low-density lipoprotein cholesterol; MAP, mean arterial pressure ; PP, pulse pressure; SBP, systolic blood pressure; TG, triglycerides, WC, waist circumference.

positive relationship of high MAP with the risk of CHD was consistent with a previous studies. ${ }^{26}{ }^{27}$ Besides, this relationship was confirmed in cohort studies from the USA and Swedish. ${ }^{57}$ However, not all studies have reported the same relationship between $\mathrm{BP}$ and the risk of $\mathrm{CHD}^{28}$; a study suggested that BP did not predict the risk of ischaemic heart disease in patients with high TG/ low HDL-C. Therefore, our study has performed mediation analysis to examine the contribution of lipid level to MAP-related CHD.
In the present analysis, we reported that $3.57 \%-21.43 \%$ of excess relative risk of CHD was mediated through the log-transformed value of TG, the log-transformed value of HDL-C and TG/HDL-C for MAP. The mediation may be explained by other previous studies on macroscopic and microscopic models. In the macroscopic model, previous studies showed that adults with both high TG and low HDL-C had increased risks of CHD. ${ }^{15}{ }^{29-31}$ In the microscopic model, the collaborative analysis of 101 studies in 2010 found a series of findings consistent with the causal 
Table 2 OR and $95 \% \mathrm{Cl}$ of $\mathrm{CHD}$ according to MAP

\begin{tabular}{lllllll}
\hline Variables & $\begin{array}{l}\text { Q1: }<84 \\
(\mathbf{n}=9438)\end{array}$ & $\begin{array}{l}\text { Q2: 84-92 } \\
(\mathbf{n}=9181)\end{array}$ & $\begin{array}{l}\text { Q3: 93-102 } \\
(\mathbf{n}=9939)\end{array}$ & $\begin{array}{l}\text { Q4: } \geq \mathbf{1 0 3} \\
(\mathbf{n}=\mathbf{1 0 4 6 2})\end{array}$ & P for trend & 1-SD increment \\
\hline Model 1 & 1 & $1.29(1.11-1.50)$ & $1.36(1.17-1.58)$ & $1.63(1.41-1.88)$ & $<0.001$ & $1.14(1.09-1.19)$ \\
Model 2 & 1 & $1.31(1.12-1.52)$ & $1.38(1.19-1.61)$ & $1.65(1.43-1.91)$ & $<0.001$ & $1.15(1.09-1.20)$ \\
Model 3 & 1 & $1.21(1.04-1.41)$ & $1.28(1.10-1.49)$ & $1.38(1.20-1.60)$ & $<0.001$ & $1.09(1.04-1.15)$ \\
\hline
\end{tabular}

Model 1: unadjusted.

Model 2: adjusted for age and gender.

Model 3: adjusted for age, gender, smoking, drinking, physical activity, family history of CHD, antihypertensive, lipid-lowering treatments and type 2 diabetes.

$\mathrm{CHD}$, coronary heart disease; MAP, mean arterial pressure.

role of TG-mediated pathways in CHD. ${ }^{17}$ In 2012, a study suggested that the risk of CHD associated with low HDL-C in women was greater than twofold to fourfold elevated depending on TG levels and elevated TG and low HDL-G with a substantially low CHD risk. ${ }^{32}$ In addition, a study has found that isolated low HDL-C or high TG levels are independently associated with CHD risk in men. ${ }^{33} \mathrm{An}$ epidemiological study indicated that TC, TG and TG/ HDL-C were independent important risk factors for CHD after adjustment for age and traditional risk factors; however, TC only TG/HDL-C but not TG was an independent predictor of CHD risk. ${ }^{16}$ However, the underlying mechanisms were unclear.

As far as we know, this is not a conventional epidemiological study to look for causal factors of CHD. The focus of this article was instead the contribution of lipid level to MAP-related CHD based on a relatively large sample size of rural population in China. These findings have shown the implication for the development of CHD prevention strategies. Moreover, the integration of evidence from genetic association studies (ie, Mendelian randomisation) may help determine the nature of this association. Standardised survey tools, training and field implementation, and adjustment for various potential confounders ensure the reliability of the analysis. Another strength of this study was that mediation analysis was performed to address whether MAP-related CHD was explained by TG, HDL-C and TG/HDL-C and calculated the proportion explained risk mediated of each mediator. Our study may help generate hypothesis that can be used to identify high-risk patients with CHD who might get benefit from better control on their BP.

However, some limitations should also be mentioned on the basis of these results. First, the main limitation of this study was that it was cross sectional and thus retrospectively identified patients with CHD, where the temporal relationship between MAP and CHD could not be assessed. First, the main limitation of the current study was that it was cross sectional, and thus patients with CHD were identified retrospectively, where the temporal relationship between MAP and CHD could not be evaluated. Second, the subjects were from only one province (Henan province) accounting for only 10\% of China's rural population, which may not be representative of the entire rural areas of China. Therefore, the current findings need to be further validated in prospective and multicultural studies. Although the current study has these limitations, the results based on a relatively large
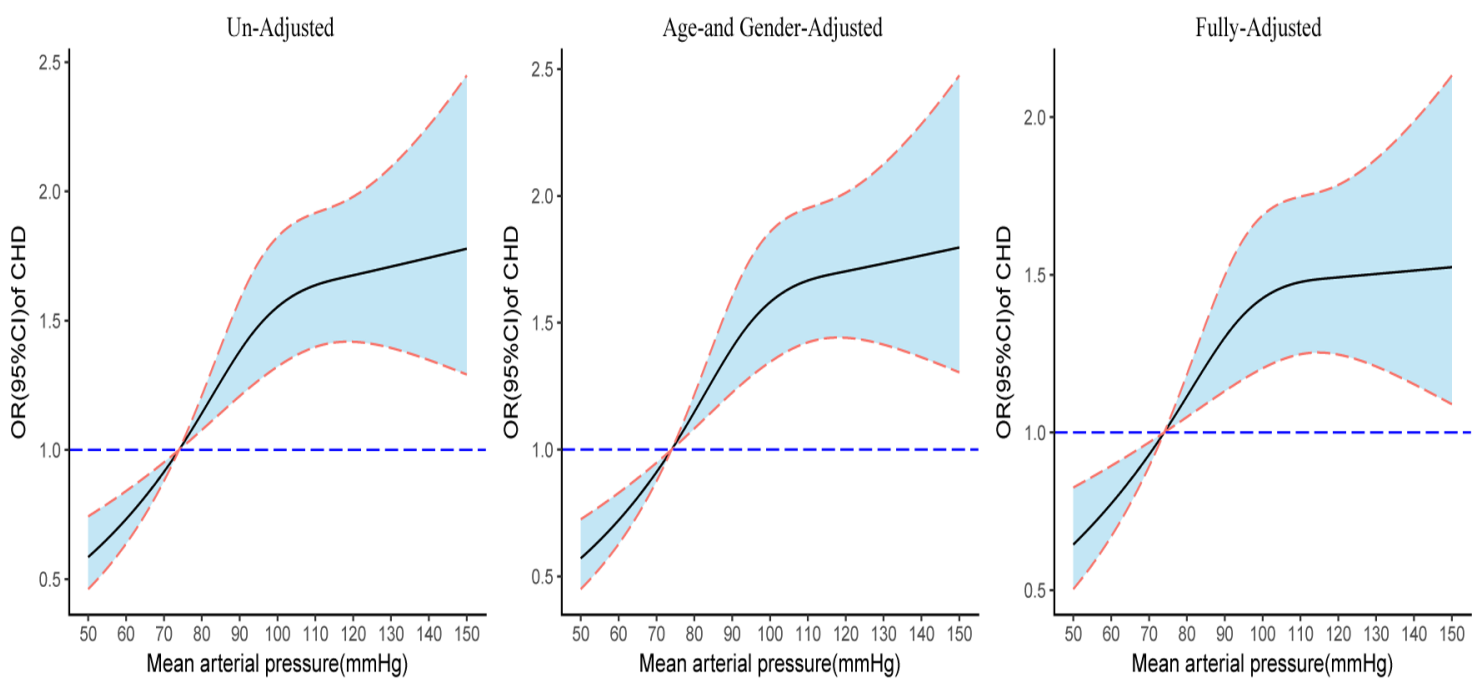

Figure $1 \mathrm{OR}$ (solid lines) and $95 \% \mathrm{Cl}$ (dashed lines) for the risk of $\mathrm{CHD}$ along with the changes of MAP from restricted cubic splines regression model. Fully-Adjusted was adjusted for age, gender, smoking, drinking, physical activity, family history of $\mathrm{CHD}$, antihypertensive, lipid-lowering treatments and Type 2 diabetes mellitus. 
A

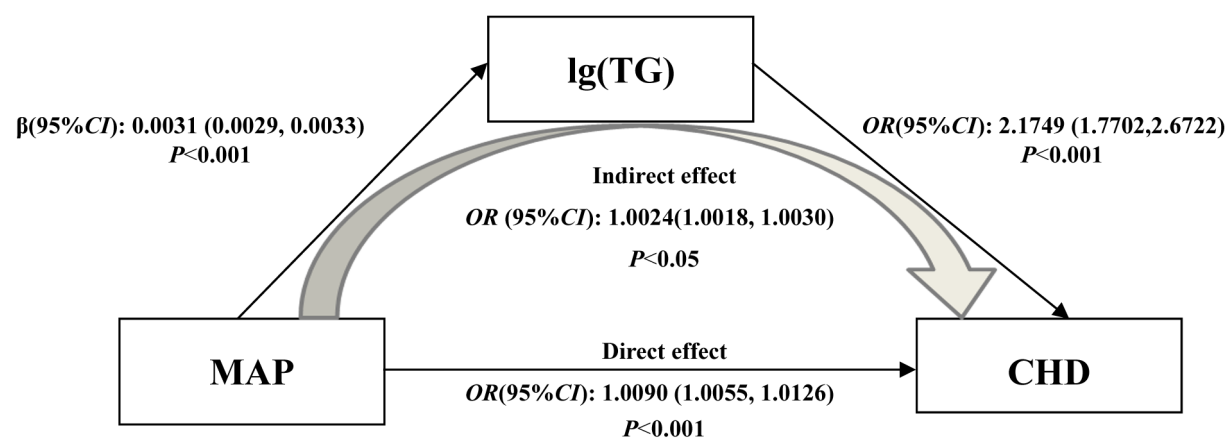

B
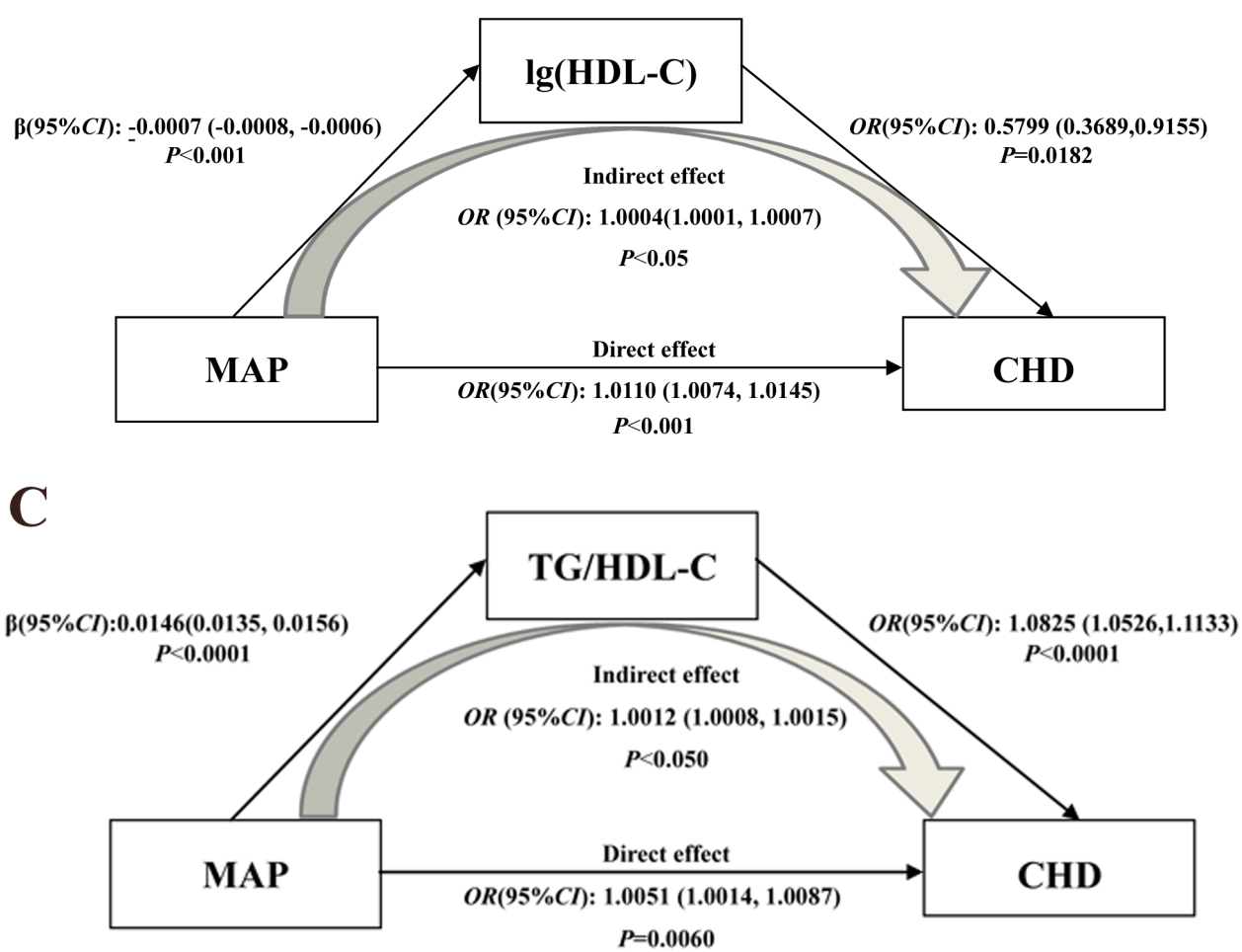

Figure 2 Mediation analysis to determine the relationship between MAP and CHD through the log-transformed value of TG (A), the log-transformed value of HDL-C (B) and TG/HDL-C (C). OR (95\% Cl; p value) of total effect was 1.0113 (1.0078 to 1.0148; 0.001). Adjusted for age, gender, smoking, drinking, physical activity, family history of $\mathrm{CHD}$, antihypertensive, lipid-lowering treatments and type 2 diabetes mellitus. CHD, coronary heart disease; HDL-C, low-density lipoprotein cholesterol; MAP, mean arterial pressure; TG, triglycerides

rural epidemiological study can, to some extent, represent the relationship between MAP and CHD in rural areas of China.

\section{CONCLUSION}

A linear dose-response relationship between MAP and CHD was first observed in Chinese rural population. Also, increased MAP was a significant marker of CHD in Chinese rural population. Simultaneously, the logtransformed value of TG was a significantly stronger mediator in the relationship. However, further prospective and multicultural researches are needed to verify these findings. Future work should focus on the clinical implications of assessment of variability in BP with serum lipids to avoid the common confounding pitfalls observed to date. In addition, more attention should be paid to the integration of evidence from genetic association studies (ie, Mendelian randomisation) that may help to determine the nature of this association.

\section{Author affiliations}

${ }^{1}$ Department of Epidemiology and Biostatistics, College of Public Health, Zhengzhou University, Zhengzhou, China

${ }^{2}$ Department of Clinical Pharmacology, School of Pharmaceutical Science, Zhengzhou University, Zhengzhou, Henan, China

${ }^{3}$ Department of Encephalopathy District, The First Affiliated Hospital of Henan University of CM, Zhengzhou, China 
${ }^{4}$ Department of health policy research, Henan Academy of Medical Sciences, Zhengzhou, China

Acknowledgements The authors would like to thank all our team members for their contributions to the epidemiological investigation. In addition, the authors would like to thank Professor Ling Wang and Dr Erhui Cai for their critical reading of the manuscript.

Contributors CJW conceived and designed the study. XZ, YQL, YHW, KH and RQT coordinated data collection. HQZ, ZYT, DQ and GYZ conducted the analyses. XZ wrote the manuscript. All coauthors critically revised the manuscript. All authors have approved the final manuscript.

Funding This research was supported by the National Key Research and Development Programme of China (Grant No. 2016YFC0900803), National Natural Science Foundation of China (Grant Nos. 81573243, 81602925 and U1304821), Henan Natural Science Foundation of China (Grant No. 182300410293), Henan Provincial Science Fund for Distinguished Young Scholars (Grant No. 164100510021), Science and Technology Innovation Talents Support Plan of Henan Province Colleges and Universities (Grant No. 14HASTIT035) and High-level Personnel Special Support Project of Zhengzhou University (Grant No. ZDGD13001). The funders had no role in the study design, data collection and analysis, decision to publish or preparation of the manuscript.

Competing interests None declared.

Patient consent for publication Not required.

Ethics approval The Henan Rural Cohort Study was approved by the Zhengzhou University Life Science Ethics Committee and was conducted according to the 1975 Declaration of Helsinki.

Provenance and peer review Not commissioned; externally peer reviewed.

Data availability statement Data are available on reasonable request.

Open access This is an open access article distributed in accordance with the Creative Commons Attribution Non Commercial (CC BY-NC 4.0) license, which permits others to distribute, remix, adapt, build upon this work non-commercially, and license their derivative works on different terms, provided the original work is properly cited, appropriate credit is given, any changes made indicated, and the use is non-commercial. See: http://creativecommons.org/licenses/by-nc/4.0/.

ORCID iD

Chongjian Wang http://orcid.org/0000-0001-5091-6621

\section{REFERENCES}

1 Clark H. Ncds: a challenge to sustainable human development. Lancet 2013;381:510-1.

2 Roth GA, Johnson C, Abajobir A, et al. Global, regional, and national burden of cardiovascular diseases for 10 causes, 1990 to 2015. J Am Coll Cardiol 2017;70:1-25.

3 GBD. 2013 mortality and causes of death Collaborators. causespecific mortality for 240 causes in China during 1990-2013: a systematic sub national analysis for the global burden of disease study 2013. Lancet 2016:251-72.

4 Chen WW, Gao RL, Liu LS, et al. China cardiovascular disease report 2017 summary. Chinese Circulation Journal 2018;33:1-7.

5 Cheng S, Gupta DK, Claggett B, et al. Differential influence of distinct components of increased blood pressure on cardiovascular outcomes: from the Atherosclerosis risk in Communities study. Hypertension 2013;62:492-8.

6 Franklin SS, Lopez VA, Wong ND, et al. Single versus combined blood pressure components and risk for cardiovascular disease. Circulation 2009;119:243-50.

7 Falkstedt D, Koupil I, Hemmingsson T. Blood pressure in late adolescence and early incidence of coronary heart disease and stroke in the Swedish 1969 conscription cohort. J Hypertens 2008;26:1313-20.

8 Boloukat RR, Ramezankhani A, Hasheminia M, et al. Impact of blood pressure, cholesterol and glucose in the association between adiposity measures and coronary heart disease and stroke among Iranian population. Clin Nutr 2017;S0261-5614:31355-9.

9 Dart AM, Kingwell BA. Pulse pressure-a review of mechanisms and clinical relevance. J Am Coll Cardiol 2001;37:975-84.

10 Miura K, Dyer AR, Greenland P, et al. Pulse pressure compared with other blood pressure indexes in compared with other blood pressure indexes in the prediction of 25-year cardiovascular and all-cause mortality rates: the Chicago heart association detection project in industry study. Hypertension 2001;38:232-7.

11 Dorjgochoo T, Shu XO, Zhang X, et al. Relation of blood pressure components and categories and all-cause, stroke and coronary heart disease mortality in urban Chinese women: a population-based prospective study. J Hypertens 2009;27:468-75.

12 Weitzman D, Goldbourt U, Dahlia W, Uri G. The significance of various blood pressure indices for long-term stroke, coronary heart disease, and all-cause mortality in men: the Israeli ischemic heart disease study. Stroke 2006;37:358-63.

13 Borghi C, Rodriguez-Artalejo F, De Backer G, et al. The association between blood pressure and lipid levels in Europe: European study on cardiovascular risk prevention and management in usual daily practice. J Hypertens 2016;34:2155-63.

14 Wakabayashi I. Associations of blood lipid-related indices with blood pressure and pulse pressure in middle-aged men. Metab Syndr Relat Disord 2015;13:22-8.

15 Lee JS, Chang P-Y, Zhang Y, et al. Triglyceride and HDL-C dyslipidemia and risks of coronary heart disease and ischemic stroke by glycemic dysregulation status: the strong heart study. Diabetes Care 2017;40:529-37.

16 Hadaegh F, Khalili D, Ghasemi A, et al. Triglyceride/HDLcholesterol ratio is an independent predictor for coronary heart disease in a population of Iranian men. Nutr Metab Cardiovasc Dis 2009;19:401-8

17 Sarwar N, Sandhu MS, Ricketts SL, et al. Triglyceride-mediated pathways and coronary disease: collaborative analysis of 101 studies. Lancet 2010;375:1634-9.

18 Rana JS, Visser ME, Arsenault BJ, et al. Metabolic dyslipidemia and risk of future coronary heart disease in apparently healthy men and women: the EPIC-Norfolk prospective population study. Int J Cardiol 2010;143:399-404.

19 Liu X, Yu S, Mao Z, et al. Dyslipidemia prevalence, awareness, treatment, control, and risk factors in Chinese rural population: the Henan rural cohort study. Lipids Health Dis 2018;17.

20 Perloff D, Grim C, Flack J, et al. Human blood pressure determination by sphygmomanometry. Circulation 1993;88:2460-70.

21 Zhou H, Li Y, Liu X, et al. Development and evaluation of a risk score for type 2 diabetes mellitus among middle-aged Chinese rural population based on the RuralDiab study. Sci Rep 2017;7:42685.

22 World Health Organization. International Statistical Classification of Diseases and Related Health Problems 10th Revision (ICD-10)WHO Version for; 2016, Chapter IX Diseases of the circulatory system(I00-199) Ischaemic heart diseases (I20- 125). Available: http://apps.who.int/classifications/icd10/browse/2016/en\#/I20-I25 [Accessed 24 Oct 2017].

23 Desquilbet L, Mariotti F. Dose-Response analyses using restricted cubic spline functions in public health research. Stat Med 2010;29:1037-57.

24 Preacher KJ, Hayes AF. SPSS and SAS procedures for estimating indirect effects in simple mediation models. Behavior Research Methods, Instruments, \& Computers 2004;36:717-31.

25 Korsiak J, Tranmer J, Day A, et al. Sleep duration as a mediator between an alternating day and night shift work schedule and metabolic syndrome among female Hospital employees. Occup Environ Med 2018;75:132-8.

26 Ettehad D, Emdin CA, Kiran A, et al. Blood pressure lowering for prevention of cardiovascular disease and death: a systematic review and meta-analysis. The Lancet 2016;387:957-67.

27 Brant LJ, Ferrucci L, Sheng SL, et al. Gender differences in the accuracy of time-dependent blood pressure indices for predicting coronary heart disease: a random-effects modeling approach. Gend Med 2010;7:616-27.

28 Jeppesen J, Hein $\mathrm{HO}$, Suadicani P, et al. High triglycerides and low HDL cholesterol and blood pressure and risk of ischemic heart disease. Hypertension 2000;36:226-32.

29 Ballantyne CM, Olsson AG, Cook TJ, et al. Influence of low highdensity lipoprotein cholesterol and elevated triglyceride on coronary heart disease events and response to simvastatin therapy in $4 \mathrm{~S}$. Circulation 2001:104:3046-51.

30 Manninen V, Tenkanen L, Koskinen P, et al. Joint effects of serum triglyceride and LDL cholesterol and HDL cholesterol concentrations on coronary heart disease risk in the Helsinki heart study. Implications for treatment. Circulation 1992;85:37-45.

31 Jeppesen J, Hein HO, Suadicani P, et al. Low Triglycerides-High high-density lipoprotein cholesterol and risk of ischemic heart disease. Arch Intern Med 2001;161:361-6.

32 Abdel-Maksoud MF, Eckel RH, Hamman RF, et al. Risk of coronary heart disease is associated with triglycerides and high-density lipoprotein cholesterol in women and non-high-density lipoprotein cholesterol in men. J Clin Lipidol 2012;6:374-81. 
33 Rana JS, Liu JY, Moffet HH, et al. Metabolic Dyslipidemia and Risk of Coronary Heart Disease in 28,318 Adults With Diabetes Mellitus and Low-Density Lipoprotein Cholesterol $<100$ mg/dl. Am J Cardiol 2015;116:1700-4. 